\title{
Die Konstruktion von Grenzen: Der Wandel einer Ausgrenzungspolitik zu einer Integrationspolitik
}

\section{Christine Leuenberger}

\begin{abstract}
Der Fall der Berliner Mauer 1989 leitete eine neue Ära der Mauern ein. Mit vermehrten Errichtungen von Grenzbarrieren stieg in den Sozialwissenschaften auch das Interesse an Border Studies, welche sich kritisch mit den Folgen neuer Grenzziehungen und Umzäunungen in einer vermeintlich globalisierten Welt auseinandersetzen. Dieser Beitrag befasst sich damit, warum Mauern und Sperren entlang nationaler Grenzen als politische Strategie bevorzugt werden und warum der Ruf nach durchlässigeren Grenzen und für mehr transnationale Zusammenarbeit lauter wird. Der Text schließt mit einem Ausblick darauf, wie angesichts aktueller globaler Bevölkerungsverschiebungen eine Ausgrenzungspolitik in eine Integrationspolitik umgewandelt werden kann.
\end{abstract}

\section{Schlagwörter}

Mauern, Migration, Grenzpolitik, Border Studies

\section{Einleitung: Ein Neues Zeitalter der Mauern ${ }^{1}$}

Mit dem Fall der Berliner Mauer im Jahr 1989 schien eine neue Ära offener geografischer Räume und einer einmaligen Mobilität zu beginnen, welche die vormals ideologisch und politisch geteilte Welt ersetzte. In den 1990er-Jahren lösten Globalisierung, Deterritorialisierung und die Öffnung von Grenzen vermeintlich Nationalismus, Regionalismus und territorial verankerte, kulturelle Identitäten $\mathrm{ab}$. Zu dieser Zeit schienen begrenzte Räume einer grenzenlosen und vernetzten Welt zu weichen. Wirtschaftliche Handelsabkommen zwischen den Mitgliedsstaaten der Europäischen Union (EU) und ähnlichen Handelsblöcken verbesserten die transnationale wirtschaftliche Vernetzung. Die Entwicklung grenzüberschreitender Regionen in Europa, Asien und den Amerikas erweckte den Eindruck, dass Nationalstaaten und deren Grenzen nicht mehr die Bedingungen globaler Vernetzungen diktierten. Diese Vision einer grenzenlosen Welt kollidierte jedoch zunehmend mit einem Wiederaufkommen von Nationalismus und Grenzschließungen. Die Ära nach 1989 brachte ein neues Zeitalter der Mauern mit sich, welches eine Ausbreitung von physischen und virtuellen Grenzen, Umgrenzungen und Grenzgebieten nach sich zog (vgl. Ohmae 1990; Vallet 2014; Bissonnette/Vallet 2020) ${ }^{2}$. Nicht nur in der westlichen Hemisphäre, sondern auch im Nahen Osten, in Afrika und in Asien wurden neue ausgrenzenden Infrastrukturen (wie Mauern, Barrieren und Zäune ${ }^{3}$ ) zunehmend errichtet.

1 Eine frühere Version von Teilen dieses Aufsatzes erschien auf englisch unter dem Titel Crumbling Walls and Mass Migration in the 21st Century (Leuenberger 2019).

2 Der Wunsch nach offenen Grenzen war nicht universell, wie die politischen Entwicklungen im Nahen Osten zeigen (siehe Leuenberger/Schnell 2020).

3 Häufig haben die Begriffe Mauern, Barrieren und Zäune politische Bedeutungen. Gegner*innen solcher Infrastrukturen benutzen häufig den Begriff Mauer (der negative Konnotationen im Zusammenhang mit diktatorischer Macht und dauerhafter Trennung hervorruft), wobei Protagonisten*innen hingegen die Bezeichnung ,Zaun“ (welcher eine vermeintlich nachbarschaftliche vorübergehende Trennung bezeichnet) verwenden. Wie Rosiere (2011) jedoch betont, heben moderne Hightechgrenzsysteme, welche Sperren mit elektronischen Überwachungssystemen 
Die unerwartete Verhärtung von Grenzen hat die Sozialwissenschaften dazu veranlasst, die soziokulturellen, politischen und wirtschaftlichen Funktionen und Auswirkungen verschiedener Grenzmechanismen zu untersuchen. Wissenschaftler*innen, die mit dem inter- und transdisziplinären Feld von Border Studies assoziiert sind, nutzten das Konzept der Grenze zunehmend als Linse, um solche Themen wie Territorialität, Grenzmanagement, Machtverhältnisse und die soziokulturelle Konstruktion von Grenzen und Grenzgebieten zu belichten (vgl. Newman 2017). Angesichts der immer komplexeren Raumdimension von Grenzen im 21. Jahrhundert hat die Soziologin Saskia Sassen dafür plädiert, Räume und Grenzen nicht nur innerhalb von Grenzgebieten, sondern auch als gesellschaftsweite soziale Praktiken, Gesetzgebungen und Diskurse zu untersuchen (vgl. Sassen 2006). Dementsprechend befinden sich Grenzmechanismen und ihre Auswirkungen in einem komplexen Netzwerk institutioneller und narrativer Praktiken, welche territoriale Imaginationen, kulturelle Identitäten und Machtverhältnisse koproduzieren können (vgl. Newman/Paasi 1998; Brunet-Jailly 2011).

Geograf*innen haben auch analysiert, wie materiale, virtuelle und symbolische Grenzen kulturelle und politische Identitäten bilden und verändern können (vgl. Falah/Newman 1995). Historiker*innen wiederum haben die Bedeutung von Grenzen für die Staats- und Nationenbildung, territoriale Souveränität und die Konstruktion nationaler Identitäten nachgezeichnet (vgl. Anderson 1983; Hobsbawn 1992). Feldexpert*innen haben sich auf Grenzziehungen und deren Auswirkungen konzentriert (vgl. Schofield et al. 2002; Biger 2008). Doch wie Wissenschaftler*innen aus den Science and Technology Studies herausgearbeitet haben, muss die Materialität von Grenzlinien, durch eine Reihe von physischen Objekten, Papieren, Karten als auch durch konzeptuelle Daten konstituiert, verstanden werden (vgl. Lynch 1994; Turnbull 1994; Leuenberger/Schnell 2010; Leuenberger 2016a; 2016b). Dieses Verbinden des Abstrakten und Greifbaren macht das aus, was Bruno Latour (1986) „unveränderliches mobiles Element“ (immutable mobile) nennt, das raumüberschreitend ist und dazu dienen kann (je nach Interaktions- und Verhandlungskontingenzen), andere, welche die Grenze nicht erfahren haben, von dessen Lage und Realität zu überzeugen. Solche Studien bestätigen, dass die Bedeutungen von Grenzen nicht fix, sondern fließend sind, und dass Grenzen, Grenzfunktionen und dementsprechend grenzüberschreitende Beziehungen in spezifischen historischen und soziokulturellen Kontexten konstituiert werden.

In Angesicht des neuen Zeitalters der Mauer nach 1989 haben Wissenschaftler*innen unterschiedliche Erklärungen für die Vermehrung verschiedener Ausgrenzungsstrategien vorgeschlagen. Saskia Sassen (2014) argumentiert, dass die Verbreitung des globalen Kapitalismus nach 1989 für viele Wohlstand und wirtschaftliche Möglichkeiten eröffnete, doch zugleich aber auch extreme soziale und wirtschaftliche Ungleichheiten hervorbrachte - sowohl innerhalb als auch zwischen Ländern. Solche Ungleichheiten haben zu einem Bauboom von Grenzbarrieren beigetragen. Einige Wissenschaftler"innen haben sogar grenzüberschreitende Ungleichheiten als den robustesten Prädiktor für den Bau von Grenzmauern benannt (vgl. auch Schäfer in diesem Band).

Darüber hinaus haben die Terroranschläge auf die Twin Towers in New York City in den Vereinigten Staaten am 11. September 2001 zu einem „Sicherheitsprimat“ (Konrad 2014) geführt. Dementsprechend wurden Grenzgebiete stets weniger als Regionen möglicher kultureller und wirtschaftlicher Integration betrachtet, sondern stets mehr als Sicherheitsbedrohungen

verbinden, den Unterschied zwischen Mauern und Zäunen auf, da diese das unbefugte Betreten gleichermaßen wirksam verhindern. 
definiert. Durch die Einrichtung neuer Grenzmechanismen soll dementsprechend die Sicherheit erhöht, der Terrorismus eingedämmt, ethnische Gewalt minimiert und illegale Einwanderung, Schmuggel und Drogenhandel unterbunden werden. Mit dem Ende des Kalten Krieges und der abnehmenden Bedrohung durch die Sowjetunion wurden dabei staatliche Ressourcen auf die Grenzsicherung umgelenkt. Gleichzeitig wurde das Paradigma des Sicherheitsprimates zu einem politischen Instrument, um den Bau „harter Grenzen“ (Vallet 2014) zu rechtfertigen was auch zunehmend zu einem profitablen Industriekomplex für Grenzsicherung wurde (vgl. Schivone 2018).

Wissenschaftler*innen behaupten auch, dass neben wirtschaftlichen Ungleichheiten und dem Aufkommen des Sicherheitsprimates die globale Verbreitung des Neoliberalismus nach dem Kalten Krieg mit politischen, ökonomischen und sozial-ethnischen Destabilisierungen einherging. In letzter Zeit hat die politische Destabilisierung des Nahen Ostens (speziell von Syrien) entscheidend zur Zunahme von Flüchtlingsströmen beigetragen. Gleichzeitig haben politisch fragile und scheiternde Regierungen in Subsahara-Afrika und anderswo die Zahl der Migrant*innen erhöht. ${ }^{4}$

Die historischen Gründe für die Fragilität dieser Nationalstaaten sind vielfältig. Sie reichen von den Folgen des Kolonialismus, des Neokolonialismus und der Einführung westlich geprägter Demokratien in politische und kulturelle Kontexte, die ihnen nicht zugänglich sind, bis hin zu unmittelbareren Ursachen wie den Auswirkungen gegenwärtiger neoliberaler Politik (vgl. Sen 1999; Owusu 2003). So sollen beispielsweise nach dem neoliberalen Diktat unterfinanzierte und schwache Nationalstaaten eine ,Politik der offenen Tür' verfolgen, die durch Deregulierung, Privatisierung und den Zufluss von ausländischem Kapital und Investitionen gekennzeichnet ist (vgl. Leuenberger/El-Atrash 2015). Eine solche Politik bereichert häufig einige lokale und internationale Eliten, während lokale Infrastrukturen, Ressourcen und $\mathrm{Hu}$ mankapital weitgehend unerschlossen bleiben (vgl. French 2015). In der Zeit der rapiden Globalisierung nach 1989 wurden oft unterfinanzierte Länder des Globalen Südens diesem neoliberalem Diktat unterworfen. Dies hat zu Ungleichheiten innerhalb und zwischen Ländern geführt, was verschiedene soziale Probleme verschärft und damit die Migration fördert.

Bereits 2002 argumentierte der Präsident der Weltbank James Wolfensohn: „The world will not be stable if we do not deal with the question of poverty. If it is not stable, we will be affected by migration, crime, drugs and terror" (Owusu 2003, S. 1667). In einer Zeit verstärkter Bevölkerungsbewegungen klingen diese Worte beunruhigend wahr. Bis 2018 wurden weltweit fast 71 Millionen Menschen gewaltsam vertrieben, dementsprechend war einer von 108 Menschen entweder Flüchtling, intern vertrieben oder asylsuchend (UNHCR o.J.). Solche Bevölkerungsbewegungen sind die massivsten seit dem Zweiten Weltkrieg und es wird erwartet, dass sie nur noch zunehmen werden. Laut Joas Estevens (2018) ist demnach das 21. Jahrhundert das Jahrhundert der Migrant*innen. Angesichts solcher Bevölkerungsbewegungen sind Befürworter*innen für die Wiederschließung der Grenzen mit jenen zusammengeprallt, die sich für offenere Grenzen einsetzen.

Im Folgenden (unter Abschnitt 2) wird zunächst darauf eingegangen, warum durchlässigere Grenzen von Befürworter*innen verfechtet werden. Auch werden die wirtschaftlichen, regulatorischen und moralischen Implikationen verschiedener Arten von Grenzpolitik aufgeführt

4 Die OECD (2017) bezeichnen funktionierende und legitime Nationalstaaten als das Fundament des internationalen politischen Systems, jedoch haben sich ,schwache' Staaten in letzter Zeit vermehrt. 
werden. In Abschnitt 3 liegt der Schwerpunkt auf den von politischen Entscheidungsträger*innen als ,best practices' bezeichneten Verfahren zum Umgang mit Bevölkerungsbewegungen. Abschnitt 4 erörtert die Notwendigkeit, nicht nur gegenwärtige, oft negativ besetzte Diskurse über Migrant*innen mit evidenzbasierten PR-Kampagnen zu ersetzen, die auf den Wert einer integrativen Grenzpolitik hinweisen, aber auch ein transnationales Paradigma fördern. Aktuelle Anlässe, wie die globale Gesundheitskrise des Jahres 2020, die durch die rasante weltweite Verbreitung des Sars-CoV-2-Virus verursacht wurde, bestätigen nur die dringende Notwendigkeit, grenzübergreifende Institutionen und Kooperation zu stärken.

\section{Ein Plädoyer für offene Grenzen}

Warum sollten wir offenere Grenzen erwägen? Politische Entscheidungsträger*innen und Akademiker"innen sind sich generell einig, dass Bevölkerungsbewegungen bleiben werden und demnach wird die Ausgrenzung von Migrant*innen dieser Tatsache nicht gerecht werden (vgl. Reuters 2016). Nicht nur politische Instabilität und wirtschaftliche Ungleichheit werden die Migration weiter vorantreiben (vgl. Black et al. 2006; Kahanec/Zimmermann 2008), sondern zunehmend auch der Klimawandel. Der Klimawandel wird Menschen durch unvorhersehbare Wettermuster, Überflutung der Küstenstädte und Nahrungs- und/oder Wasserknappheit und dadurch ausgelöste Konflikte vertreiben (vgl. Cramer et al. 2018; IOM 2019). Wie Paul Rogers hervorhebt,

„existing migration pressures stemming mainly from economic pressures are already being exacerbated by the impact of climate change and this will intensify greatly. [...] [S]ecuritizing the problem in terms of a ,close the castle gates' mentality simply cannot work in a globalized and interconnected world and is a futile response" (2017, S. 4).

Da Migration daher wohl eines der bestimmenden Themen des 21. Jahrhunderts sein wird, werden immer häufiger Forderungen nach durchlässigeren Grenzen laut. So weist Philippe Legrain $(2014 ; 2016)$ zum Beispiel darauf hin, dass die meist offene Grenzpolitik der USA von der Zeit nach dem Bürgerkrieg bis nach dem Ersten Weltkrieg eine Schlüsselrolle für ihren wirtschaftlichen Erfolg spielte. Andererseits hätte die restriktivere Grenzpolitik in den 1960er Jahren schätzungsweise zwölf Millionen undokumentierte Immigrant*innen mit sich gebracht, deren individuelle Rechte nicht nur begrenzt sind, aber sie sind auch von potenzieller Abschiebung bedroht. Eine solch restriktive Politik hatte nicht nur rechtliche, sondern auch wirtschaftliche Auswirkungen. Die USA gibt nun mehr für die Grenzüberwachung aus als für alle anderen Strafverfolgungsbehörden auf Bundesebene zusammen (vgl. Planas 2014).

Außerdem werden die aus dem 19. Jahrhundert stammenden Vorstellungen von souveränen und ethnisch homogenen Nationalstaaten zunehmend zu einem Anachronismus, trotz deren wichtiger Funktion, die Rechte von Bürger*innen zu sichern (vgl. Leuenberger 2013). Solche Homogenitäts- und Souveränitätsvorstellungen fördern Kulturalismus, Nationalismus und Ethnozentrismus, die nicht mit der „Glokalisierung“ (der Verschmelzung des Lokalen und Globalen und der Entstehung hybrider soziokultureller Phänomenen) moderner Gesellschaften in Einklang stehen (Bryman 1999, S. 261). In einem globalen Zeitalter, in dem Güter, Dienstleistungen und Bevölkerungen stets in Bewegung sind, wurde der Ruf nach transnationalen Regulierungsinstitutionen entsprechend lauter. Wie Julie Mostov hervorhebt: „Given the spillover effects of actions across the globe and the deep interconnections of global capitalism, it makes sense to reexamine the boundaries of political association within and across existing units 
and consider new transnational architectures of decision-making “ (2018, S. 124). Eine solche transnationale Architektur würde transnationale Organisationen mit sich bringen, die sich besser mit grenzüberschreitenden Fragen und Angelegenheiten wie Menschen- und Arbeitsrechten, Gesundheitssicherheit und internationalem Handel und Politik befassen könnten.

Da es weltweit nun jedoch 193 Länder (was fast einer Verdreifachung der Anzahl von Ländern seit 1945 entspricht) und (bis 2019) rund 70 ,harten Grenzen` gibt (vgl. Karasz 2019) und dies in jüngster Zeit mit einer Zunahme an ethnonationalen Diskursen einhergeht, klingen Forderungen nach offeneren Grenzen immer bedeutungsloser. Für Politiker*innen scheint der Begriff der offenen Grenzen in der Tat zu einer politischen Landmine geworden zu sein (vgl. Swanson 2016). Während einige Wirtschaftswissenschaftler*innen und politische Entscheidungsträger*innen (vgl. Galbraith 1979; Ohmae 1990; Legrain 2016; National Academies of Sciences, Engineering, and Medicine 2017a) die Vorteile weicherer Grenzen herausgestellt haben, hat der transnationale Aufstieg rechter Politik zunehmend immigrant*innenfeindliche Positionen und harte Grenzen begünstigt. Im Folgenden setze ich mich mit eben solchen nationalistischen Tendenzen auseinander.

\subsection{Grenzpolitik und die neue rechte Orientierung}

Politische Tendenzen zugunsten härterer Grenzen sind in letzter Zeit zunehmend gestärkt worden. Als Ungarns populistischer Premierminister Viktor Orbán 2015 den Bau eines ungarischen Grenzzauns ankündigte, um angeblich Wirtschaftsmigrant*innen oder Terroristen *innen auszugrenzen, bezeichnete der Menschenrechtskommissar des Europarats dies als „schlecht beraten“ (Feher 2015). Die EU verurteilte Orbáns Grenzpolitik und ermutigte ihn, stattdessen europäische Werte zu fördern (Europäisches Parlament 2015). Nach dem Zweiten Weltkrieg gab es in Europa im Allgemeinen ein Einvernehmen, dass offenere Grenzen, die wirtschaftliche Integration der EU, Demokratie und Frieden sowie Menschen- und Asylrechte die Basis des europäischen Projektes sind. Diese Werte unterlagen der progressiven, liberalen und egalitären Sichtweise der EU. Folglich erhielt die EU 2012 den Friedensnobelpreis dafür, dass sie einen Kontinent der Kriege und Konflikte in einen Kontinent des Friedens verwandelt hat. Im Jahr 2015 war sich das politische Establishment Europas daher noch weitgehend darin einig, dass der Bau von Zäunen und Mauern weder europäische Werte fördert noch der Herausforderung vermehrter Bevölkerungsbewegungen gerecht wird.

Dieser Konsens nach dem Kalten Krieg, liberale Werte zu fördern, die Menschenrechte zu respektieren und zu einer Politik der offenen Tür zu ermutigen, ist jedoch zugunsten der aufkommenden neuen rechten Orientierung in Europa und den Vereinigten Staaten zunehmend bedroht. Im April 2016 warnte US-Präsident Barack Obama noch vor „a creeping emergence of the kind of politics that the European project was founded to reject: an us-versus-them mentality that tries to blame our problems on the other" (The White House 2016). Doch in Europa waren rechtspopulistische Bewegungen auf dem Vormarsch. Am 23. Juni 2016 war das liberale, zentristische Establishment schockiert über die Entscheidung der britischen Öffentlichkeit, sich in der Brexit-Abstimmung aus der EU zurückzuziehen. Populistische, gegen das Establishment gerichtete, einwander*innenfeindliche und nationalistische Stimmungen und Rhetoriken schienen die Antwort für diejenigen zu sein, die sich durch die Globalisierung zurückgelassen fühlten. Der Ruf nach einer Verstärkung der Grenzen wurde immer lauter. 
In den Vereinigten Staaten schürte der damalige republikanische Präsidentschaftskandidat Donald Trump während des Wahlkampfes 2016 seine Anhänger*innen an, indem er „Build That Wall“ rief (in Anspielung auf die Verstärkung der amerikanisch-mexikanischen Grenzmauer). Die Anti-Immigrant*innen-Rhetorik sollte die Herzen und Seelen eines Volkes gewinnen. Die Präsidentschaftswahl von Trump am 8. November 2016 signalisierte den zunehmenden Wechsel zu einer rechtsextremen und populistischen Politik.

Kritiker*innen verweisen auf verschiedene Gründe für den Aufstieg rechtspopulistischer Politik, die von einer Gegenreaktion auf die Globalisierung und den Neoliberalismus, wirtschaftliche Ungleichheit, Unsicherheit und Stagnation bis hin zu einer aufkommenden Klasse wirtschaftlicher Verlierer*innen der globalen neoliberalen Ordnung reichen. Für viele kritische Beobachter*innen ist es die Zunahme der wirtschaftlichen Ungleichheit, die sich zermürbend auf Gesellschaft und Demokratie ausgewirkt hat (Sassen 2014). Solch steigende wirtschaftliche Ungleichheit geht stets mehr einher mit populistischen Führer*innen, welche Negativität und Emotionen wie Angst und Wut ausnutzen und die Rhetorik von ,wir gegen die anderen " als einen rhetorischen Tropus benutzen (Leuenberger/Schnell 2010). In einer Zeit, in der globale Belange innovative Lösungen verlangen, werden daher kurzfristige Abhilfemaßnahmen wie härtere Grenzen - für festgefahrene und langfristige Probleme - immer häufiger eingesetzt.

\subsection{Grenzpolitik: Ökonomie, transnationale Regulierung und moralische Imperative}

Wissenschaftler*innen der Border Studies haben die aktuelle Grenzpolitik und dessen Auswirkungen auf Wirtschaft, Politik, Kultur und Gesellschaft untersucht (vgl. Vallet 2014; Jones 2019). Aufgrund der gegenwärtigen Vorherrschaft neoliberaler Marktpolitik (vgl. Polanyi 1947; Brenner et al. 2002) und der Notwendigkeit politischer Akteur*innen, die Sprache des hegemonischen neoliberalen Diskurses zu sprechen (vgl. Owusu 2003), sind wirtschaftliche Argumente im Hinblick auf die gegenwärtige Grenzpolitik ein Versuch, politische Trennlinien zu überwinden. Was sind demzufolge wirtschaftlichen Argumente gegen harte Grenzen?

Wirtschaftswissenschaftler*innen, wie der klassische Wirtschaftstheoretiker John Kenneth Galbraith (1979), haben argumentiert, dass offene Grenzen wirksame Lösungen zur Förderung des Wirtschaftswachstums, zur Bekämpfung globaler Ungleichheit und zur Verringerung weltweiter Armut sind. Nationale Grenzen behindern in der Tat das wirtschaftliche Wachstum. Sie begrenzen Humankapital an Orten, an denen menschliche Fähigkeiten ungenutzt bleiben, und sie zementieren die Ungleichheit an Ort und Stelle. Entsprechend warb Galbraith für die wirtschaftlichen Vorteile unbegrenzter Einwanderung und offener Grenzen:

„Migration, we have seen, is the oldest action against poverty. It selects those who most want help. It is good for the country to which they go; it helps to break the equilibrium of poverty in the country from which they come. What is the perversity in the human soul that causes people so to resist so obvious a good?" (1979, S. 136).

Die Migration kann dem Migrant*innen-Entsendeland zugute kommen. In der Tat könnten offenere Grenzen Armut viel wirksamer bekämpfen als die Entsendung von Entwicklungshilfe. Die Höhe der Überweisungen, die Migrant"innen in ihre Heimatländer schicken, übersteigt bei Weitem die bereitgestellten Mittel der Entwicklungshilfe und könnten so zu deren Stabilität und wirtschaftlichem Wohlergehen beitragen (vgl. z. B. Legrain 2014; National Academies of Sciences, Engineering, and Medicine 2017a; Openborders 2017). 
Studien haben auch gezeigt, dass Migrant*innen, die sich frei bewegen können, weniger geneigt sind, sich dauerhaft neu anzusiedeln. Stattdessen kehren sie mit größerer Wahrscheinlichkeit in ihr Entsendeland zurück und bringen Fähigkeiten und Vermögenswerte mit, die zum lokalen Lebensstandard beitragen können (vgl. Kahanec/Zimmermann 2008; IZA 2015). Tatsächlich ergab eine Gallup-Umfrage von 2010, dass bei offenen Grenzen nur etwa 14 Prozent der erwachsenen Weltbevölkerung dauerhaft in ein anderes Land ziehen möchte (Caplan/Naik 2015). Folglich neigt eine restriktive Einwanderungspolitik dazu, fehlzuschlagen, da Arbeitnehmer*innen dann auf illegale Mittel zurückgreifen, um in das Empfänger*innenland einzureisen und nicht mehr in ihr Entsendeland zurückzukehren. Andererseits würde eine weniger restriktive Politik zirkuläre Bewegungen fördern (vgl. dazu Nail in diesem Band).

Migration kann auch dem Empfänger*innenland zugute kommen. Migrationsgegner*innen gehen oft davon aus, dass die Größe der Wirtschaft feststehe und Migrant*innen den bereits dort Beschäftigten Arbeitsplätze wegnehmen. Die Wirtschaft jedoch wächst in der Folge von Migration. Migrant*innen nehmen nicht nur Arbeitsplätze an, sondern schaffen sie auch. Sie neigen - mehr als Nichtimmigrant*innen - dazu, neue Unternehmen zu öffnen und sind im Allgemeinen durch ihre Arbeitsamkeit, Motivation und einen Innovationsgeist ausgezeichnet. Ihre Ausgaben erhöhen auch die Nachfrage, was sowohl die Wirtschaft als auch das Bruttoinlandsprodukt langfristig ankurbelt. Darüber hinaus unterscheiden sich ihre Fähigkeiten tendenziell von denen der einheimischen Arbeitnehmer*innen und stehen dabei meist nicht in direkter Konkurrenz, sondern ergänzen sich eher (vgl. Legrain 2014). Migrant*innen sind in der Regel auch jung - ein entscheidendes Merkmal in Ländern mit einer zunehmend alternden Erwerbsbevölkerung. Zusätzlich zahlen sie in der Regel Steuern, haben niedrige medizinische Kosten und leisten Beiträge zur Altersvorsorge (vgl. Ku 2009). Migration kann damit sowohl den Entsende- als auch den Aufnahmeländern zugute kommen (vgl. Vollmer/Düvell in diesem Band).

Zusätzlich zu diesen wirtschaftlichen Argumenten wurde darauf hingewiesen (vgl. Kysel 2016; MPI o.J.; OHCHR o.J.), dass globale Wirtschaften bestimmte regulatorische und moralische Herausforderungen mit sich bringen, denen das jetzige System noch nicht gerecht wird. Zum Beispiel tendieren Institutionen dazu, in ihrer Reichweite eher stets noch national statt transnational zu agieren. Gewerkschaften sind ein typisches Beispiel dafür. In einer transnationalen Wirtschaft werden national organisierte Gewerkschaften in ihrer Reichweite und Relevanz zunehmend eingeschränkt. Dasselbe gilt für Menschenrechte, wenn diese nicht von einem souveränen Staat bestimmt werden. Entsprechend weist Legrain darauf hin, dass der Staat „in the twenty-first century will have to get used to managing the rights and status of nationals who are outside its territory, and aliens who are in its territory - in other words, to dealing with populations on the move“ (2014, S. 319). Die Funktionsfähigkeit eines transnationalen und global vernetzten Wirtschaftssystems hängt von der Mobilität der Arbeitskräfte ab, dennoch sind Regelungen weiterhin auf nationale Ebenen beschränkt.

Eine globale Wirtschaft bringt auch moralische Herausforderungen mit sich. Laut Legrain (2014) kommt eine Wirtschaft, in der Grenzen für Waren, Dienstleistungen und eine privilegierte globale Bürgerschaft ,weich`sind, aber für die sozioökonomisch Benachteiligten ,hart bleiben, einer Form von globaler Apartheid gleich. Zusätzlich erläutert er, dass Integration zunehmende Vielfalt mit sich bringt, was menschliche Fähigkeiten, komplexe Probleme zu lösen, steigert. Migrant*innen bringen zum Beispiel auch „different skills, varied views, diverse experiences and a zeal for self-improvement that combine with the talents of local people 
to boost innovation, productivity and economic growth" (ebd., S. 19). In der Tat wurden Intel, Google und viele Unternehmen im Silicon Valley von Einwanderer*innen gegründet; ein Drittel der Amerikaner*innen, die in den letzten sieben Jahren einen Nobelpreis für Physik gewonnen haben und fast 50 Prozent der Doktoranden*innen in den Natur- und Ingenieurwissenschaften sind im Ausland geboren (National Science Foundation 2020). Langfristig gesehen hat Migration daher einige Vorteile. Daher sind für Alex Tabarrok harte Grenzen „one of the world's greatest moral failings but the opening of borders is the world's greatest economic opportunity“ (Tabarrok 2015).

\section{Bewährte Praktiken: Integrative Taktiken in verschiedenen nationalen Kontexten}

Im Jahr 2015 erklärte der UN-Hochkommissar für Flüchtlinge António Guterres (der später UN-Generalsekretär wurde): „For an age of unprecedented mass displacement, we need an unprecedented humanitarian response and a renewed global commitment to tolerance and protection for people fleeing conflict and persecution“ (UN News 2015). Im selben Jahr wies UN-Generalsekretär Ban Ki-moon darauf hin, dass „the components needed for a comprehensive solution are at hand, but they need to be implemented!“ (UN News 2016). Beispielhafte Lösungen, um mit Bevölkerungsbewegungen umzugehen, gibt es verschiedene. ${ }^{5}$ Einige Länder haben bereits unterschiedliche integrative Taktiken erfolgreich umgesetzt.

Zum Beispiel hat Deutschland gewisse bewährte Praktiken im Hinblick auf die Integration von Migrant*innen umgesetzt. Das Land hat im Jahr 2015 1,1 Millionen Migrant*innen aufgenommen und integriert (obwohl diese Zahl 2016 zurückgegangen ist; vgl. DESTASIS o.J.). Damals lobte die Regierung des ehemaligen US-Präsidenten Obama Deutschland nicht nur dafür, dass es (neben Schweden) die meisten Flüchtlinge in Europa aufnahm, sondern auch für die zahlreichen Bundes- und Landesmittel, die der Integrationsförderung zugeschoben wurden. So entwickelte beispielsweise das Ministerium für Migration und Flüchtlinge (zusammen mit der Bundesagentur für Arbeit) ein integratives Flüchtlingsverwaltungssystem (vgl. Deutsches Bundesamt für Migration und Flüchtlinge 2016). Ziel war es, den Asyl- und Integrationsprozess zu beschleunigen, die Sicherheitsüberprüfungen zu verbessern und die schnelle kulturelle und sprachliche Integration von Migrant*innen zu fördern.

Politische Entscheidungsträger*innen und Thinktanks haben auch darauf hingewiesen, dass eine kulturelle und wirtschaftliche Integration in das Gastland auch Gefühle der Entfremdung und Entrechtung unter Neuzuwanderer*innen vermindert und damit eine wirksame Maßnahme zur Terrorismusbekämpfung darstellt (vgl. Schiffauer et al. 2017). Die Förderung der Integration von Migrant*innen ist angesichts der ergrauenden Erwerbsbevölkerung und der schrumpfenden Bevölkerungszahlen gerade auch für Deutschland wirtschaftlich vorteilhaft. Folglich kann die Integration von Migrant*innen dazu beitragen, den demografischen Rückgang umzukehren und die Renten der alternden Babyboomer zu bezahlen. Während das Engagement der deutschen Regierung für die Integration von Migrant*innen zu jener Zeit in einigen Beziehungen als beispielhaft hervorgehoben wurde, hat der Aufstieg rechtsgerichteter

5 Konkrete Vorschläge mit vermehrter Migration umzugehen, sind z.B. die Ausweitung der Anforderungen für legale Einwanderung, die Eröffnung konsularischer Außenposten, sodass humanitäre Visa dort und nicht im Gastland beantragt werden können, und die Ausweitung von Umsiedlungsprogrammen. Auch sollte das internationale Wirtschaftswesen und die Entwicklungshilfe so reformiert werden, dass Ursachen der Migration minimiert werden (vgl. French 2015; Goodwin-Gill/Sazak 2015). 
Parteiprogramme (wie jene der AfD - Alternative für Deutschland) und die Kritik an der damaligen Offenen-Tür-Politik von Bundeskanzlerin Angela Merkel zunehmend zu einer populären und politischen Gegenreaktion geführt. Die nachfolgenden Entwicklungen haben die politische Landschaft nachgehend in Richtung einer härteren Grenzpolitik verändert.

Der Staat Israel ist ein weiteres Beispiel, das zeigt, wie die Integration einer vielfältigen Bevölkerung nationalen Interessen dienen kann. Trotz Israels ausschließender Politik gegenüber Palästinenser*innen und der Marginalisierung arabischer Israelis innerhalb der israelischen Gesellschaft, kann der Staat dennoch als Vorbild für eine erfolgreiche Einwanderungspolitik dienen, wenn auch nur für Menschen jüdischer Herkunft (vgl. Ministry of Immigrant Absorption o.J.). Obwohl das israelische Rückkehrgesetz wegen seiner Diskriminierung von Nichtjüd*innen politisch höchst umstritten ist, erlaubt es Menschen jüdischer Herkunft, die Staatsbürgerschaft in Israel zu beanspruchen.

In den 1990er Jahren absorbierte Israels flexible Wirtschaft ankommende Migrant*innen und blühte dadurch auf, was den Hightechsektor und eine Kultur des Internetunternehmergeistes förderten. Ein entscheidender Faktor für die erfolgreiche Integration von Neuzuwanderer*innen ist das Engagement des Staates für deren umzügliche Integration. Das Ministry for Immigrant Absorption (Ministerium für die Aufnahme von Einwanderer*innen) weist Migrant*innen auf Ressourcen hin, die für eine erfolgreiche Integration benötigt werden. Migrant*innen erhalten Hilfe bei der Wohnungs- und Arbeitssuche, kostenlosen Sprachunterricht und Begrüßungspakete, um sie im ersten Jahr nach ihrer Ankunft finanziell zu unterstützen. Sie erlangen ebenfalls schnell das Bürger*innenrecht, was ihnen ein Gefühl der gesellschaftlichen Zugehörigkeit vermittelt. Doch trotz der angebotenen Integrationshilfe wird von den Migrant*innen nicht erwartet, dass sie ihr kulturelles Erbe aufgeben. Die Integrationspolitik in Israel zeigt daher ebenfalls, wie entscheidend das Engagement des Staates für die erfolgreiche Integration von Immigrant*innen sein kann (vgl. Legrain 2014).

Derartige Best-Practice-Beispiele sind jedoch nicht nur auf den Globalen Norden beschränkt. Angesichts der großen Zahl von Migrant*innen, die in Ländern im Globalen Süden landen, bietet die ugandische Regierung auch ein Modell für eine beispielhafte Integrationspolitik. Uganda wird oft als Flüchtlingsoase bezeichnet, da es Asylsuchende durch eine Strategie der Eigenständigkeit und einen Aktionsplan zur Armutsbekämpfung zur Integration und zu langfristig tragfähigen Lebensbedingungen verhilft (vgl. Dryden-Peterson/Hovil 2003; Meyer 2006; Byaruhanga 2016). Ugandas Asylpolitik gibt Migrant*innen das Recht auf Arbeit und Bewegungsfreiheit. Ebenso ermöglicht Ugandas Siedlungsmodell für Flüchtlinge, dass Migrant*innen in Siedlungen und nicht in Lagern wohnen können. Diese Politik erlaubt es Migrant*innen, „für sich selbst zu fischen“ (Easton-Calabria 2016, S. 14). Um dies zu erreichen, weist die Regierung neuen Migrant*innen Grundstücke für ihre eigene landwirtschaftliche Nutzung und Selbstversorgung zu. Ziel ist es dabei, durch Eigenständigkeit (statt Fürsorge) Entwicklung zu ermöglichen. Obwohl das ugandische Flüchtlingsmodell auch oft hinter seinen Absichten zurückbleibt, wird es oft als ein wahres Modell für Flüchtlingsverwaltung gelobt (vgl. Kigozi 2017).

Wie die obigen Beispiele zeigen, sind bewährte Praktiken bekannt - ob sie in Deutschland, Israel oder Uganda ausgeführt werden. Angesichts dessen, was im Jahr 2016 oft als eine ,Flüchtlingskrise' beschrieben wurde, ermutigten damals politische Entscheidungsträger*innen zur Förderung des Austauschs, um bewährte Integrationspraktiken zu ermitteln. In der Tat wird die Entwicklung von Strategien, um mit demografischen Veränderungen und Bevölke- 
rungsbewegungen umzugehen, eine der wichtigsten Herausforderungen dieses Jahrzehntes bleiben.

\section{Neue Diskurse und neue Geschichten}

Angesichts der oben diskutierten Vorteile einer offeneren Grenzpolitik ist es entscheidend, den öffentlichen Diskurs über Themen wie Flüchtlinge, Migrant*innen, Grenzen und deren Auswirkungen zu belichten (siehe dazu auch Lehner in diesem Band). Diese Diskurse sind oft höchst politisiert und daher ist es wichtig, mehr evidenzbasierte Botschaften zu diesen Themen zu vermitteln. Wie Legrain betont:

„The key message [...] is that policymakers and practitioners should stop considering refugees as a ,burden' to be shared, but as an opportunity to be welcomed. With a suitable upfront investment and wise polices, welcoming refugees can yield substantial economic dividends“ (2016, S. 9).

Das Overseas Development Institute (ODI) fordert, dass staatliche und nichtstaatliche Institutionen sich vermehrt anstrengen, oft weit verbreiteten einwanderungsfeindlichen Botschaften mit mehr einwanderungsfreundlichen zu begegnen. Demnach sollten die Vorteile von Migration mehr betont werden.

„European politicians and the wider public need to start seeing migrants and refugees as a valuable resource rather than a problem. By executing policies that limit the agency of migrants and asylum seekers, host countries are missing out on the economic benefits of migration and new arrivals are being robbed of their capacity to support themselves. It doesn't have to be like this. But policy change is unlikely to happen if public and political support isn't there - support that must be built on a recognition of the benefits of migration“ (ODI 2016, S. 7).

Dennoch werden in sensationslüsternen Bildern, die in den Nachrichten und im Internet kursieren, über die Grenzen strömende Migrant*innen gezeigt. Die Darstellung solcher Bilder, zusammen mit Kommentaren von politisch rechtsorientierten Politiker*innen, hat die Stimmung in der Bevölkerung zunehmend gegen eine offenere Grenzpolitik gewendet. Solche Darstellungen betonen die angebliche Andersartigkeit von Flüchtlingen und Migrant"innen. Wie jedoch die Geschichte des 20. Jahrhunderts zeigt, sind Darstellungen von Flüchtlingen unweigerlich mit bestimmten politischen Agenden verbunden. So wurden beispielsweise politische Flüchtlinge, die während des Kalten Krieges von der Sowjetunion in den Westen flohen, für ihre Integrität und ihr Heldentum angesehen. Sowjetische Dissident*innen, wie z.B. Alexander Solzhenitsyn, wurden als „public intellectuals, moral thinkers, samizdat writers, artists standing up for freedom of artistic expression against political oppression" anerkannt (Pupavac 2008, S. 273). Während daher damals Flüchtlinge (wegen der politischen Konkurrenz zwischen den Supermächten) ideologische Werte besaßen (vgl. Chimni 1998), verloren sie diese nach dem Ende des Kalten Krieges. Heutzutage werden Flüchtlinge eher als verletzliche, feminisierte und traumatisierte Opfer dargestellt, denen es an politischer Handlungsfähigkeit mangelt und die von Behörden betreut werden müssen. Solche Vorstellungen gehen vermehrt mit weniger gesetzlichen Rechten für Flüchtlinge einher. Gleichzeitig haben Politiker*innen zunehmend Fremdenfeindlichkeit und eine Angst vor anderen begünstigt, indem sie Migrant*innen mit Terrorist*innen, Trojanischen Pferden und Schlangen gleichsetzten (eine Metapher, das beispielsweise von US-Präsident Donald Trump verwendet wurde). Doch statt solche erniedrigen- 
den Diskurse zu zirkulieren, sollte vermehrt auf die Leistungen und die Menschlichkeit von Migrant*innen eingegangen werden. Solche positiven Diskurse sollten Moral und Ethik, aber auch Menschen- und Asylrechte hervorheben. Dies kann oft besser durch Erzählungen betont werden. Gerade bei solch politisch umstrittenen Themen, bei denen wissenschaftliche Evidenz politische Ideologien oft nicht übertrumpfen kann, sind Erzählungen eine wirksame Weise, Wissen zu vermitteln (vgl. National Research Council 2012; Cairney et al. 2016; National Academies of Sciences, Engineering, and Medicine 2017b).

Moralische, ethische und menschenrechtliche Argumente für eine offenere Grenzpolitik gibt es zu genügend (vgl. Mostov 2008; Legrain 2014; vgl. auch Banse in diesem Band). Sie befassen sich mit solchen Themen wie der Notwendigkeit der menschlichen Solidarität (vgl. Jones 2019) und der Willkür von Grenzziehungen (vgl. Anderson 1993), bis hin zur Erfordernis, Menschen-und Asylrechte zu wahren. Einige Wissenschaftler*innen haben sich dabei auch auf das afrikanische Konzept des $u b u n t u$ berufen, um über Begriffe wie Zusammengehörigkeit und Verantwortung gegenüber anderen nachzudenken (vgl. Oppenheim 2012). Die Bedeutung von ubuntu wird häufig durch das Erzählen von Geschichten ausgeführt. In vielen afrikanischen Ländern ist das Erzählen von Geschichten ein wirksames pädagogisches Hilfsmittel, um Wissen und Weisheiten zu vermitteln (vgl. Chinyowa 2014). Dementsprechend erklärte Nelson Mandela die Bedeutung von ubuntu wie folgt,

„A traveler through a country would stop at a village and he didn't have to ask for food or for water. Once he stops, the people give him food, entertain him [...] The question therefore is: Are you going to do so in order to enable the community around you to be able to improve? “ (Oppenheim 2012, S. 169).

Laut Anthony Giddens (2002) hat die Globalisierung die Welt in ein globales Dorf verwandelt und wie wir nun mit Grenzen, im und um das Dorf, umgehen werden, wird unweigerlich die Dorfstruktur von morgen prägen. Grenzpolitik wird demnach stets mehr zu einem Kaleidoskop, durch das sich unterschiedliche und sich stets verändernde gesellschaftliche Werte und Diskurse sowie auch politische Bestrebungen erkennen lassen.

\section{Aus aktuellem Anlass: Eine globale Gesundheitskatastrophe und ein erneuter Appell für grenzüberschreitende Zusammenarbeit}

Nach dem Zweiten Weltkrieg waren sich Entscheidungsträger*innen im Allgemeinen einig, dass transnationale und supranationale Institutionen und Leitungsgremien wie die UN (United Nations), die WHO (World Health Organisation) und die EU (European Union) notwendig sind, um den Weltfrieden zu bewahren und globale Probleme und Angelegenheiten gemeinsam anzugehen. Der wachsende Transnationalismus und die Globalisierung während des 20. Jahrhunderts brachten die schwindende Souveränität der Nationalstaaten mit sich (vgl. Brown 2010), was folglich auch den vermehrten Grenzkonsolidierungen und dem neuen Nationalismus zugrunde liegt. Diese Verhärtung von bürokratischen und materiellen Grenzen fand statt, trotzdem es in einer globalisierten Welt nicht möglich ist, Entwicklung, Sicherheit, Umweltschutz und Menschenrechte als exklusiv nationale Probleme zu verstehen (vgl. Evans 2005). Doch auch Themen wie Migration und Grenzpolitik werden nun wieder vermehrt als nationale und nicht als transnationale Angelegenheiten behandelt. Die Stärkung und Konsolidierung einer „transnationalen Architektur zur Entscheidungsfindung” (Mostov 2008, S. 124) ist entscheidend, um Fragen bezüglich Bevölkerungsbewegungen, politischer Instabilitäten, 
wirtschaftlicher Ungleichheiten oder Klimawandel effektiver angehen zu können. Im Hinblick auf Migration würde das mehr internationale Zusammenarbeit und eine einheitlichere Migrations- und Asylpolitik erfordern (vgl. CMS 2017).

Angesichts der globalen Gesundheitspandemie von 2020, die eine rasante, grenzüberschreitende und weltweite Verbreitung des virulenten Sars-CoV-2-Virus mit sich brachte, ist eine „transnationale Architektur zur Entscheidungsfindung“ (vgl. Mostov 2008) noch dringlicher geworden. Doch die Epidemie führte zu einer noch nie dagewesenen weltweiten Schließung der Grenzen, um die Ausbreitung des Sars-CoV-2-Virus zu stoppen. Die langfristigen sozialen, wirtschaftlichen und politischen Implikationen solcher groß angelegten und transnationalen Grenzschließungen verleihen den Border Studies eine erneute Wichtigkeit. Wie sich eine solch einschneidende Pandemie auf erneuten Nationalismus und Grenzschließungen, aber auch auf die Möglichkeit erneuter Paradigmen für internationale Zusammenarbeit in Zukunft auswirken wird, ist noch nicht abzusehen (vgl. Center for Migration Studies 2020; Garrett 2020; Igoye 2020; Shachar 2020). Die Folgen dieser Pandemie machen jedenfalls den Mangel an transnationaler Zusammenarbeit und gegenseitigem Vertrauen sowie das Versagen der Politik und Wissenschaftsdiplomatie in einer Zeit deutlich, in der sich Politiker"innen zunehmend in eine nationalistische Rhetorik zurückziehen und gleichzeitig Grenzen verhärten (vgl. Barker 2020; Beacock 2020; Colglazier 2020; Rafiq 2020; Roig 2020; Wilson Center 2020). In dieser Zeit ist der Ruf nach transnationaler Zusammenarbeit zur Bekämpfung einer globalen Pandemie dringender denn je. Transnationale Institutionen - sei es in der Wissenschaft oder in der Politik - sind in solch dunklen Zeiten zu Fackelträger*innen geworden. Die Dringlichkeit einer einheitlichen globalen Reaktion auf eine solche Bedrohung hat in der Tat zu einer beispiellosen Zusammenarbeit innerhalb der Naturwissenschaften geführt (vgl. Apuzzo/Kirkpatrick 2020). Folglich könnte die Dringlichkeit einer solchen globalen Bedrohung dominante Paradigmen für grenzüberschreitende Zusammenarbeit und Grenzpolitik möglicherweise unabsehbar verändern.

Im Jahr 1968 machte der Astronaut William Anders eine Aufnahme des Planeten Erde vom Weltall aus. Es wurde zur einflussreichsten Umweltfotografie, die je aufgenommen wurde. Die außergewöhnliche Fotografie zeigte den im Weltraum schwebenden Planeten Erde, auf dem keine Grenzen sichtbar waren. Diese Aufnahme veränderte unsere Vorstellungen über die Biosphäre, den Klimawandel, globale Gesundheit und menschliche Verbundenheit (vgl. WHO 2003). Die Epidemie von 2020 ist ein weiterer historischer Moment, der zeigt, dass politische Grenzen auch tödliche Viren nicht aufhalten können. Im Hinblick auf Fragen der globalen Gesundheitssicherheit ist es demnach dringlicher denn je, Paradigmen der Grenzpolitik zu überdenken und transnationale Zusammenarbeit in Wissenschaft und Politik zu fördern.

\section{Aus dem Englischen von Ines Bergfort und Christine Leuenberger}

\section{Weiterführende Literatur}

Vallet, Elisabeth (Hrsg.) (2014): Borders, Fences and Walls: State of Insecurity? Burlington: Ashgate.

Jones, Reece (Hrsg.) (2019): Open Borders: In Defense of Free Movement. Athens: University of Georgia Press.

Sassen, Sassen (2014): Expulsions: Brutality and Complexity in the Global Economy. Cambridge: Harvard University Press.

Legrain, Phillipe (2014): Immigrants: Your County Needs Them. Princeton: Princeton University Press. 


\section{Literaturverzeichnis}

Apuzzo, Matt/Kirkpatrick, David D. (2020): Covid-19 Changed How the World Does Science, Together. The New York Times, 1.4.2020. www.nytimes.com/2020/04/01/world/europe/coronavirus-science-resea rch-cooperation.html, 20.5.2020.

Anderson, Benedict (1983): Imagined Communities: Reflections on the Origin and Spread of Nationalism. London: Verso.

Byaruhanga, Catherine (2016): Why Uganda Is So Good for Refugees. BBC, 12.5.2016. www.bbc.com/ne ws/av/world-africa-36284316/why-uganda-is-so-good-for-refugees, 20.5.2020.

Barker, Vanessa (2020): The Social Borders of Covid-19: From Social Darwinism to Social recognition. University of Oxford, Border Criminologies Blog. www.law.ox.ac.uk/research-subject-groups/centre-cri minology/centreborder-criminologies/blog/2020/04/social-borders, 20.5.2020.

Beacock, Ian (2020): Germany gets it. The New Republic, 1.4.2020. https://newrepublic.com/article/1571 12/germany-gets-coronavirus, 20.5.2020.

Biger, Gideon (2008): The Boundaries of Israel - Palestine Past, Present, and Future: A Critical Geographical View. In: Israel Studies 13, H. 1, S. 68-93.

Bissonnette, Andréanne/Vallet, Elisabeth (Hrsg.) (2020): Borders and Border Walls: In-Security, Symbolism, Vulnerabilities. London: Routledge.

Black, Richard/Natali, Claudia/Skinner, Jessica (2006): Equity \& Development World Development Report 2006. Background Papers: Migration and Inequity. www.rrojasdatabank.info/wir2006/black.pdf, 13.7.2020.

Brenner, Neil/Theodore, Nik (2002): Cities and the Geographies of Actually Existing Neoliberalism. In: Antipode 34, H. 3, S. 349-379.

Brown, Wendy (2010): Walled States, Waning Sovereignty. Cambridge: Zone Books.

Brunet-Jailly, Emmanuel (2011): Special Section: Borders, Borderlands and Theory: An Introduction. In: Geopolitics 16, H. 1, S. 1-6.

Bryman, Alan (1999): Global Disney. In: Slater, David/Taylor, Peter J. (Hrsg): The American Century. Oxford: Blackwell, S. 261-272.

Caplan, Bryan/Naik, Vipul (2015): A Radical Case for Open Borders. In: Benjamin Powell (Hrsg.): The Economics of Immigration: Market-Based Approaches, Social Science, and Public Policy. Oxford: Oxford University Press, S. 180-209.

Cairney, Paul/Oliver, Kathryn/Wellstead, Adam (2016): To Bridge the Divide between Evidence and Policy: Reduce Ambiguity as Much as Uncertainty. In: Public Administration Review 76, H. 3, S. 399-402.

Center for Migration Studies (2020): Covid-19 Migration-Related Developments. www.cmsny.org/cms-initiatives/migration-covid/, 20.5.2020.

Chinyowa, Kennedy C. (2014): The Sarungano and Shona Storytelling: An African Theatrical paradigm. In: Journal in Theatre and Performance 21, H. 1, S. 18-30.

CMS (Center for Migration Studies of New York) (2017): International Migration Policy Report: Responsibility Sharing for Large Movements of Refugees and Migrants in Need of Protection. www.cmsny.org/publications/scalabrini-policy-report-2017/, 20.5.2020.

Colglazier, William E. (2020): Response to the COVID-19 Pandemic: Catastrophic Failures of the sciencePolicy Interface. Science Diplomacy, 4.9.2020. www.sciencediplomacy.org/editorial/2020/response-covid-19-pandemic-catastrophic-failures-science-policy-interface, 20.5.2020.

Chimni, B. S. (1998): The Geopolitics of Refugee Studies: A View from the South. In: Journal of Refugee Studies 11, H. 4, S. 350-374.

Cramer, W./Guiot, J/Fader, M./Garrabou, J./Gattuso, J-P./Iglesias, A./Lange, M.A./Lionello, P./Llasat, M.C./Paz, S./Peñuelas, J./Snoussi, M./Toreti, A./Tsimplis, M. N./Xoplaki, E. (2018): Climate change and interconnected risks to sustainable development in the Mediterranean. In: Nature: Climate Change 8, S. 972-980.

Dryden-Peterson, Sarah/Hovil, Lucy (2003): Local Integration as a Durable Solution: Refugees, Host Populations and Education in Uganda. UNHCR Policy Development and Evaluation Service Working Paper 93. www.unhcr.org/3f8189ec4.pdf, 14.7.2020.

Easton-Calabria, Evan (2006): „Refugees asked to fish for themselves“: The Role of Livelihoods Trainings for Kampala's Urban Refugees. UNHCR: Policy Development and Evaluation Service. www.refworld.or g/pdfid/56bda31c4.pdf, 20.5.2020.

European Parliament (2015): Situation in Hungary: European Parliament Resolution of 10 June 2015 on the Situation in Hungary (2015/2700 RSP). www.europarl.europa.eu/doceo/document/TA-8-2015-0227 _EN.html, 21.4.2020.

Evans, Tony (2005): International Human Rights Law as Power/Knowledge. In: Human Rights Quarterly 27, H. 3, S. 1046-1068. 
Estevens, Joas (2018): Migration crisis in the EU: developing a framework for analysis of national security and defense strategies. In: Comparative Migration Studies 6, H. 28, S. 1-21.

Falah, Ghazi/Newman, David (1995): The spatial manifestation of threat: Israelis and Palestinians seek a „good“ border. In: Political Geography 14, H. 8, S. 689-706.

Feher, Margit (2015): Europe's Existence Threatened by Influx of Migrants, Says Hungary's Orban. The Wall Street Journal, 26.7.2015. www.wsj.com/articles/europes-existence-threatened-by-influx-of-migran ts-says-hungarys-orban-1437827281, 19.6.2020.

French, Howard W. (2015): The Plunder of Africa: How Everybody Holds the Continent Back. Foreign Affairs, Juli/August 2015. www.foreignaffairs.com/reviews/2015-06-16/plunder-africa, 21.4.2020.

Garrett, Terence M. (2020): Covid-19. Wall building, and the effects on Migrant Protection Protocols by the Trump administration: the spectacle of the worsening human rights disaster on the Mexico-U.S. border. In: Administrative Theory \& Praxis 42, H. 2, S. 240-248.

Galbraith, John K. (1979): The Nature of Mass Poverty. Cambridge: Harvard University Press.

German Federal Office for Migration and Refugees (2016). Der Mensch im Blick - Sicherheit geben. Chancen ermöglichen. Wandel leben. www.bamf.de/DE/Startseite/startseite-node.html, 20.5.2020.

DESTASIS Statistisches Bundesamt (o.J.): Die Datenbank des Statistischen Bundesamtes. www.genesis.dest atis.de/genesis/online, 20.5.2020.

Giddens, Anthony (2002): Runaway World: How Globalization is Reshaping Our Lives. London: Routledge.

Goodwin-Gill, Guy S./Sazak, Selim C. (2015): Footing the Bill. Foreign Affairs, 29.7.2015. www.foreignaf fairs.com/articles/africa/2015-07-29/footing-bill, 21.4.2020.

The Guardian (2016): The Observer View on Immigration - Observer editorial: Europe's response to refugees has been pitiful, 15.5.2016. www.theguardian.com/commentisfree/2016/may/14/observer-view -refugees-immigrants-europe-response, 20.5.2020.

Hjelmgaard, Kim (2018): From 7 to 77: There's been an explosion in building border walls since World War II. USA TODAY, 28.5.2018. www.usatoday.com/story/news/world/2018/05/24/border-walls-berlin -wall-donald-trump-wall/553250002/, 20.5.2020.

Igoye, Agnes (2020): Migration and Immigration: Uganda and the Covid-19 Pandemic. In: Journal Public Integrity. DOI: 10.1080/10999922.2020.1753383.

IOM (The International Organization for Migration) (2019): Climate Change and Migration in Vulnerable Countries A snapshot of least developed countries, landlocked developing countries and small island developing States. www.environmentalmigration.iom.int/climate-change-and-migration-vulnerable-countries-snapshot-least-developed-countries-landlocked, 20.5.2020.

IZA World of Labor (2015): The Impact of migration on sending and receiving countries. wol.iza.org/ news/the-impact-of-migration-on-sending-and-receiving-countries, 20.5.2020

Jones, Reece (Hrsg.) (2019): Open Borders: In Defense of Free Movement. Athens: University of Georgia Press.

Kahanec Martin/Zimmermann, Klaus F. (2008): International Migration, Ethnicity, and Economic Inequality. IZA Discussion Paper 3450. http://ftp.iza.org/dp3450.pdf, 20.5.2020

Karasz, Palko (2019): Fact Check: Trump's Tweet on Border Walls in Europe. The New York Times, 17.1.2019. www.nytimes.com/2019/01/17/world/europe/fact-check-trump-border-walls.html, 20.5.2020.

Kigozi, David (2017): The Reality behind Uganda's Refugee Model. Refugees Deeply, 30.5.2017. www.newsdeeply.com/refugees/community/2017/05/30/the-reality-behind-ugandas-glowing-reput ation, 20.5.2020.

Kysel, Ian M. (2016): Promoting the Recognition and Protection of the Rights of All Migrants Using a Soft-Law International Migrants Bill of Rights. In: Journal on Migration and Human Security 4, H. 2 , S. 29-44.

Konrad, Victor (2014): Borders, Bordered Lands and Borderlands: Geographical States of Insecurity between Canada and the United States and the Impacts of Security Primacy. In: Vallet, Elisabeth (Hrsg.): Borders, Fences and Walls: State of Insecurity? Burlington: Ashgate, S. 85-102.

$\mathrm{Ku}$, Leighton (2009): Health Insurance Coverage and Medical Expenditures of Immigrants and NativeBorn Citizens in the United States. In: American Journal Public Health 99, H. 7, S. 1322-1328.

Latour, Bruno (1986): Visualization and Cognition: Drawing Things Together. In: Kuklick, Henrika (Hrsg.): Knowledge and Society Studies in the Sociology of Culture Past and Present, Vol 6. Greenwich: Jai Press, S. 1-40.

Legrain, Phillipe (2014): Immigrants: Your Country Needs Them. Princeton: Princeton University Press.

Legrain, Phillipe (2016): Refugees Work: A Humanitarian Investment That Yields Economic Dividends. www.opennetwork.net/wp-content/uploads/2016/05/Tent-Open-Refugees-Work_V13.pdf, 20.5.2020. 
Leuenberger, Christine (2016a): Maps as Politics: Mapping the West Bank Barrier. In: Journal of Borderland Studies 31, H. 3, S. 339-364.

Leuenberger, Christine (2016b): First we take Berlin then we take Jerusalem: The Geopolitics of Mapping Divided Cities and their Separation Walls. In: Geoforum Perspektiv 27, S. 14-32.

Leuenberger, Christine/El-Atrash, Ahmad (2015): Building a Neo-liberal Palestinian State under Closure: The Economic and Spatial Implications of Walls and Barriers. In: Economic Sociology European Electronic Newsletter 16, H. 2, S. 21-31.

Leuenberger, Christine/Schnell, Izhak (2010): The Politics of Maps: Constructing National Territories in Israel. In: Social Studies of Science 40, H. 6, S. 803-842.

Leuenberger, Christine/Schnell, Izhak (2020): The Politics of Maps: Cartographic Constructions of Israel/Palestine. Oxford: Oxford University Press.

Lynch, Michael (1994): Representation is Overrated: Some Critical Remarks about the Use of the Concept of Representation in Science Studies. In: Configurations 2, H. 1, S. 37-49.

OECD (Organization for Economic Co-operation and Development) (2008): Concepts and Dilemmas of State Building in Fragile Situations: From Fragility to Resilience. www.oecd.org/development/governanc epeace/conflictandfragility/docs/41100930.pdf, 20.5.2020.

Meyer, Sarah (2006): The „Refugee Aid and Development“ Approach in Uganda: Empowerment and SelfReliance of Refugees in Practice. UNHCR The UN Refugee Agency Policy Development and Evaluation Service Research 131. www.unhcr.org/4538eb172.pdf, 20.5.2020.

Ministry of Immigrant Absorption (o.J.). www.moia.gov.il/English/Pages/default.aspx, 20.5.2020.

Mostov, Julie (2008): Soft Borders: Rethinking Sovereignty and Democracy. London: Palgrave Macmillan.

MPI Migration Policy Institute (o.J). www.migrationpolicy.org/programs/migration-information-source/sp ecial-issue-migration-and-human-rights, 20.5.2020.

National Academies of Sciences, Engineering and Medicine (2017a): The Economic and Fiscal Consequences of Immigration. Washington: The National Academies Press.

National Academies of Sciences, Engineering, and Medicine (2017b): Communicating Science Effectively: A Research Agenda. Washington: The National Academies Press.

National Research Council (2012): Using Science as Evidence in Public Policy. Washington: The National Academies Press.

National Science Foundation (2020): NSB SEI Foreign Born One Pager 2020. www.nsf.gov > nsb > sei > one-pagers > Foreign-Born, 24.10.2020.

Newman, David (2017): Borders, boundaries, and borderlands. In: The International Encyclopedia of Geography. https://onlinelibrary.wiley.com/doi/abs/10.1002/9781118786352.wbieg1039, 20.5.2020.

Newman, David/Paasi, Anssi (1998): Fences and neighbors in the postmodern world: boundary narratives in political geography. In: Progress in Human Geography 22, H. 2, S. 186-207.

OHCHR (United Nations Human Rights Office of the High Commissioner) (o.J.): „Migration and Human Rights“. www.ohchr.org/EN/Issues/Migration/Pages/MigrationAndHumanRightsIndex.aspx, 20.5.2020.

Ohmae, Kenichi (1990): The Borderless World: Power and Strategy in the Interlinked Economy. New York: Harper.

Openborders (o.J.): Benefits to immigrant-sending countries. https://openborders.info/benefits-to-immigrant-sending-countries/, 20.5.2020.

Oppenheim, Claire E. (2012): Nelson Mandela and the Power of Ubuntu. In: Religions 3, S. 369-388.

Overseas Development Institute (ODI) (2016): Journeys to Europe: The Role of Policy in Migrant Decision Making. www.odi.org/publications/10317-journeys-europe-role-policy-migrant-decision-making, 20.5.2020.

Owusu, Francis (2003): Pragmatism and the gradual Shift from Dependency to Neoliberalism: The World Bank, African Leaders and Development Policy in Africa. In: World Development 31, H. 10, S. 16551672.

Polanyi, Karl (1947): Our Obsolete Market Economy: „Civilization must find a new thought pattern“. In: Commentary 3, S. 109-117.

Planas, Roque (2014): 16 Reasons Why Opening Our Borders Makes More Sense Than Militarizing Them. Huffington Post, 2.9.2014. www.huffpost.com/entry/open-borders_n_5737722, 20.5.2020.

Pupavac, Vanessa (2008): Refugee Advocacy, Traumatic Representations and Political Disenchantment. In: Government and Opposition 43, H. 2, S. 270-292.

Rafiq, Samah (2020): Open Borders: scapegoat in Covid-19 Response. YaleGlobal Online, 9.4.2020. https://yaleglobal.yale.edu/content/open-borders-scapegoat-covid-19-response, 20.5.2020.

Reeves, Richard V. (2016): In Defense of Immigrants: Here's Why America Needs Them Now More Than Ever. Brookings, 17.5.2016. www.brookings.edu/opinions/in-defense-of-immigrants-heres-why-america -needs-them-now-more-than-ever/, 20.5.2020. 
Reuters (2016): Global refugee crisis is here to stay, says humanitarian chief David Miliband, 19.1.2016. www.reuters.com/article/us-europe-migrants-refugees-miliband-idUSKCNOUX2AR, 20.5.2020.

Rogers, Paul (2017): Climate Change, Migration and Security. Oxford Research Group, 31.7.2017. www.oxfordresearchgroup.org.uk/climate-change-migration-and-security, 20.5.2020.

Roig, Alexis (2020): Science Diplomacy against the Pandemic. International Science Council, 30.3.2020. www.ingsa.org/covidtag/covid-19-commentary/scitech-webinar-1/, 20.5.2020.

Rosiere, Stephane (2011): Teichopolitics: The Politics of Border Closure. In: Si Somos Americanos. In: Revista de Estudios Transfronterizoa XI, H. 1, S. 151-193.

Sassen, Saskia (2006): Territory, Authority, Rights: From Medieval to Global Assemblages. Princeton: Princeton University Press.

Sassen, Saskia (2014): Expulsions: Brutality and Complexity in the Global Economy. Cambridge: Harvard University Press.

Schiffauer, Werner/Eilert, Anna/Rudloff, Marlene (Hrsg.) (2017): So schaffen wir das - eine Zivilgesellschaft im Aufbruch. Bielefeld: transcript.

Schivone, Gabriel (2018): The Border-Security-Industrial Complex: A Conversation with Todd Miller. Los Angeles Review of Books, 03.10.2018. www.lareviewofbooks.org/article/the-border-security-industrial -complex-a-conversation-with-todd-miller/, 14.7.2020.

Schofield, Clive/Newman, David/Drysdale, Alasdair/Allison-Brown, Janet (Hrsg.) (2002): The Razor's Edge: International Boundaries and Political Geography. London: Kluwer Law International.

Sen, Amartya (1999): Development as Freedom. Oxford: Oxford University Press.

Shachar, Ayelet (2020): Borders in the time of COVID-19. Max-Planck-Gesellschaft, 4.4.2020. www.mpg. de/14650555/borders-in-the-time-of-covid-19, 20.5.2020.

Swanson, Ana (2016): Opening up borders: An idea economists tend to love and politicians detest. The Washington Post, 14.10.2016. www.washingtonpost.com/news/wonk/wp/2016/10/14/why-economists-1 ove-and-politicians-detest-the-idea-of-opening-the-borders-to-lots-more-immigrants/, 20.5.2020.

Tabarrok, Alex (2015): The Case for Getting Rid of Borders - Completely. The Atlantic, 10.10.2015. www.theatlantic.com/business/archive/2015/10/get-rid-borderscompletely/409501/, 20.5.2020.

Turnbull, David (with a contribution from Helen Watson with the Yulgu Community at Yirrkala) (1994): Maps are Territories: Science is an Atlas. Chicago: University of Chicago Press.

UN News (2015): UN warns of „record high“ 60 million displaced amid expanding global conflicts, 19.6.2015. www.un.org/apps/news/story.asp?NewsID=51185\#.VaZk3TV7yZU, 20.5.2020.

UN News (2016): Risks of Inaction Are Considerable, Says Ban, Urging New Compact on Refugees and Migrants, 9.5.2016. www.refugeesmigrants.un.org/risks-inaction-are-considerable-says-ban-urgingnew-compact-refugees-and-migrants, 20.5.2020.

UNHCR the UN Refugee Agency (o.J.): Refugee Statistics. www.unrefugees.org/refugee-facts/statistics/, 20.5.2020.

Vallet, Elisabeth (Hrsg.) (2014): Borders, Fences and Walls: State of Insecurity? Burlington: Ashgate.

WHO (World Health Organization) (2003): Global climate change and health: an old story writ large, 27.7.2003. www.who.int/globalchange/environment/en/chapter1.pdf?ua=1, 22.4.2020.

Wilson Center (2020): Reports from North America's Borders: Experts React to New COVID-19 Travel Restrictions, 26.3.2020. www.wilsoncenter.org/article/reports-north-americas-borders-experts-react-ne w-covid-19-travel-restrictions, 20.5.2020.

The White House - Office of the Press Secretary (2016): Remarks by President Obama in

Address to the People of Europe, 25.4.2016. www.obamawhitehouse.archives.gov/the-press-office/2016/04/25/remarks-president-obama-address-people-europe, 20.5.2020. 